\title{
Oesophageal dysmotility, delayed gastric emptying and autonomic neuropathy correlate to disturbed glucose homeostasis
}

\author{
B. Ohlsson • O. Melander • O. Thorsson • R. Olsson • \\ O. Ekberg • G. Sundkvist
}

Received: 18 August 2005 / Accepted: 10 May 2006 / Published online: 11 July 2006

(C) Springer-Verlag 2006

\begin{abstract}
Aims/hypothesis Among diabetic patients, glucose homeostasis may be affected by abnormal gastrointestinal motility and autonomic neuropathy. This study analysed whether oesophageal dysmotility, delayed gastric emptying or autonomic neuropathy affect glucose homeostasis.

Materials and methods Oesophageal manometry and gastric emptying scintigraphy were performed in 20 diabetic patients. Heart-rate variation during deep breathing (expiration/inspiration $[E / I]$ ratio) and continuous subcutaneous
\end{abstract}

These results were presented as an oral presentation at the EASD meeting in Athens 2005.

\author{
B. Ohlsson $(\bowtie)$ \\ Department of Clinical Sciences, Gastroenterology Division, \\ Malmö University Hospital, Lund University, \\ Malmö, Sweden \\ e-mail: bodil.ohlsson@med.lu.se \\ O. Melander \\ Department of Clinical Sciences, \\ Diabetes and Endocrinology Division, \\ Malmö University Hospital, Lund University, \\ Malmö, Sweden \\ O. Thorsson \\ Department of Clinical Physiology, Malmö University Hospital, \\ Lund University, \\ Malmö, Sweden \\ R. Olsson · O. Ekberg \\ Department of Medical Radiology, Malmö University Hospital, \\ Lund University, \\ Malmö, Sweden \\ G. Sundkvist \\ Department of Clinical Sciences, \\ Diabetes Epidemiology and Neuropathy Division, \\ Malmö University Hospital, Lund University, \\ Malmö, Sweden
}

glucose concentrations for a period of $72 \mathrm{~h}$ were also monitored in the same patients.

Results Oesophageal dysmotility was found in eight of 14 patients. Eleven of 20 patients had delayed gastric emptying (abnormal gastric emptying half-time $\left[T_{50}\right]$ ) and nine of 18 had an abnormal E/I ratio. Complaints of abdominal fullness were predictive of delayed gastric emptying. A low peristaltic speed of the oesophagus was associated with impaired $T_{50}\left(r_{s}=-0.67 ; p=0.02\right)$. One hour after breakfast, subcutaneous glucose levels decreased in patients with delayed gastric emptying but continued to rise in those with normal emptying. Consequently, the median glucose level $2.5 \mathrm{~h}$ after breakfast was lower in the former (9.1 [4.2-12.5] vs $14.3[11.2-17.7] \mathrm{mmol} / \mathrm{l} ; p<0.05)$. Glucose fluctuations during the $72 \mathrm{~h}$ were significantly higher in patients with an abnormal $E / I$ ratio than in those with a normal $E / I$ ratio (coefficient of variation: 41 [46-49] vs $28[27-34] \%$; $p=0.008$ ).

Conclusions/interpretation Abdominal fullness predicted delayed gastric emptying that was associated with diminished glucose uptake after breakfast. Low oesophageal peristaltic speed was associated with slow gastric emptying whereas parasympathetic neuropathy was associated with increased glucose variations.

Keywords Autonomic neuropathy · CGMS .

Continuous glucose monitoring system .

Diabetes mellitus · Gastrointestinal symptoms .

Gastroparesis · Oesophageal dysmotility
Abbreviations
CGMS continuous glucose monitoring system
E/I expiration/inspiration ratio
$T_{50} \quad$ gastric emptying half-time 


\section{Introduction}

Although symptoms suggestive of gastrointestinal dysmotility poorly correlate with gastrointestinal disturbances [1], gastrointestinal dysmotility is a well-known complication of diabetes mellitus [2]. Gastrointestinal dysmotility is believed to affect glycaemic control but the mechanisms of this influence are unclear. An animal model suggests that autonomic neuropathy contributes to impaired glycaemic control [3].

Our hypothesis is that not only delayed gastric emptying but also other gastrointestinal complications impair glycaemic control. This study sought to define specific symptoms that identify upper gastrointestinal dysmotility, and to evaluate the relationships between glucose homeostasis and oesophageal dysmotility, delayed gastric emptying and autonomic neuropathy.

\section{Subjects, materials and methods}

\section{Subjects}

This study was performed in a prospective manner according to the Helsinki Declaration and was approved by the Ethics Committee of Lund University. All patients gave written informed consent before entering the study.

At the diabetes clinic at Malmö University Hospital, the first 20 consecutive patients complaining of symptoms suggestive of disturbances in the gastrointestinal tract were invited to join the current study that comprised: (1) a questionnaire with 16 symptom questions (loss of appetite, swallowing disturbances, meal-related cough, early satiety, nausea, vomiting, weight loss, abdominal fullness, bloating, regurgitation, constipation, diarrhoea, evacuation incontinence, decreased postprandial glycaemia, symptomatic postprandial hypoglycaemia, postprandial perspiration); (2) gastric emptying scintigraphy $(n=20)$; (3) oesophageal manometry $(n=14)$; (4) autonomic nervous function $(n=18)$; and (5) continuous subcutaneous glucose monitoring over $72 \mathrm{~h}(n=20)$. All patients were insulin-treated (17 had type 1 diabetes and three had type 2 diabetes). None were on medication affecting intestinal motility or the autonomic nervous system.

\section{Examinations}

Standardised oesophageal manometry was performed with the patient sitting in the upright position and the peristaltic speed was calculated. Each patient performed ten swallows. The lower oesophagal sphincter was identified during a slow pull-through in the mid-expiratory phase. Inadequate relaxation of the low oesophageal sphincter, uncoordinated oesophageal contractions or hypo/hypercontractions were considered as oesophageal dysmotility [4].

Gastric emptying (solid food) was assessed [5] and a gastric emptying half-time $\left(T_{50}\right)>2 \mathrm{SD}$ from that of healthy controls $(=70 \mathrm{~min})$ was considered to be delayed gastric emptying [6].

Respiratory rate interval variation during deep breathing (expiration/inspiration $[E / I]$ ratio) was used as a test of autonomic nerve function and an age-related $E / I$ ratio below -1.64 SD was considered abnormal [7].

Using a continuous glucose monitoring system (CGMS) (Mini Med, Sylmar, CA, USA), subcutaneous glucose levels were continuously monitored for $72 \mathrm{~h}$ [8]. The CV of glucose concentration was defined as $(\mathrm{SD} /$ mean $) \times 100$ (\%). On the second day, after an overnight fast, the patients took their usual insulin injection at the start of a fibre-rich breakfast and subcutaneous glucose levels were followed during the following $4 \mathrm{~h}$ until lunch. The breakfast consisted of oatmeal porridge, cooked from $35 \mathrm{~g}$ fibre porridge oats, $150 \mathrm{~g} 0.1 \%$ fat milk and $17 \mathrm{~g}$ mashed apple together with a slice of rye bread containing $9 \%$ fibre with $5 \mathrm{~g}$ of $40 \%$ fat margarine and $10 \mathrm{~g}$ of $17 \%$ fat cheese.

Statistical analysis

Results are expressed as median and interquartile ranges (IQR). The Mann-Whitney $U$-test and Fisher's exact test were used to calculate differences between groups, and Spearman's correlation test was used to evaluate correlations; $p<0.05$ was considered significant.

\section{Results}

Gastrointestinal dysmotility

Eleven of 20 patients had delayed gastric emptying. There were no differences in sex, age, disease duration or $\mathrm{HbA}_{1 \mathrm{c}}$ between patients with and without delayed gastric emptying (Table 1). $T_{50}$ was inversely related to age $\left(r_{s}=-0.46 ; p=0.05\right)$ but did not correlate with $\mathrm{HbA}_{1 \mathrm{c}}$ $\left(r_{s}=0.00 ; p=1.00\right)$ or duration of diabetes $\left(r_{s}=0.18 ; p=0.60\right)$. There was no difference in median $T_{50}$ between the nine patients with an abnormal $E / I$ ratio and the nine patients whose $E / I$ ratio was normal (102 [44-248] vs 117 [26-240] $\min ; p=0.96$ ).

Eight out of 14 patients showed dysmotility of the oesophagus (Table 1). There was no correlation between oesophageal dysmotility and gastric emptying or the $E / I$ ratio (data not shown). However, there was a negative correlation between mean peristaltic speed of the oesophagus and $T_{50}\left(r_{s}=-0.67 ; p=0.02\right)$. 
Table 1 Distribution of investigated parameters in patients with normal and delayed gastric emptying, respectively

\begin{tabular}{|c|c|c|c|}
\hline Parameter & Normal gastric emptying $(n=9)$ & Delayed gastric emptying $(n=11)$ & $p$ \\
\hline$T_{50}(\min )$ & $35.0(23.5-44.5)$ & $224.5(116.5-328.3)$ & $<0.001$ \\
\hline Sex (female/male) & $3 / 6$ & $7 / 4$ & 0.37 \\
\hline Age (years) & $58.0(47.0-59.5)$ & $51.0(44.0-56.0)$ & 0.20 \\
\hline BMI & $23.1(19.1-28.3)$ & $28.0(22.3-29.0)$ & 0.41 \\
\hline Duration of diabetes (years) & $27.0(22.0-46.0)$ & $33.0(24.0-44.0)$ & 0.64 \\
\hline $\mathrm{HbA}_{1 \mathrm{c}}(\%)$ & $7.6(6.5-8.4)$ & $6.9(6.3-7.6)$ & 0.37 \\
\hline$E / I$ ratio (SD from expected) & $-1.4(-2.2$ to -1.3$)$ & $-1.8(-2.1$ to -0.62$)$ & 0.89 \\
\hline \multicolumn{4}{|l|}{ Oesophageal functions } \\
\hline Dysmotility (\%) & 40 & 67 & 0.58 \\
\hline A-peristaltic swallowing (\%) & $0(0-55)$ & $20(0-40)$ & 0.70 \\
\hline Mean amplitude contractions $(\mathrm{mmHg})$ & $60(26-64)$ & $59(28-75)$ & 0.80 \\
\hline Simultaneous contractions $(\%)$ & $0(0-10)$ & $20(0-30)$ & 0.15 \\
\hline Mean peristaltic speed $(\mathrm{cm} / \mathrm{s})$ & $6.5(5.3-7.6)$ & $3.8(3.2-4.6)$ & 0.008 \\
\hline LOS pressure $(\mathrm{mmHg})$ & $5.0(0-13.8)$ & $15(7.5-20)$ & 0.15 \\
\hline \multicolumn{4}{|c|}{ Continuous subcutaneous glucose registrations } \\
\hline Baseline glucose $(\mathrm{mmol} / \mathrm{l})$ & $8.0(6.7-10.8)$ & $8.7(3.8-10.0)$ & 0.55 \\
\hline Highest glucose (mmol/l) & $17.6(14.1-18.7)$ & $13.4(12.5-16.6)$ & 0.20 \\
\hline Lowest glucose $(\mathrm{mmol} / \mathrm{l})$ & $11.1(3.7-15.0)$ & $6.8(4.0-12.7)$ & 0.66 \\
\hline Time to highest glucose (h) & $1.9(1.2-2.6)$ & $2.1(1.2-3.5)$ & 0.66 \\
\hline $\mathrm{CV}$ of glucose $(\%)$ & $42.3(28.8-48.6)$ & $36.4(30.5-53.4)$ & 0.88 \\
\hline
\end{tabular}

All data are given as median (interquartile range). $p<0.05$ was considered significant

For patients investigated with oesophageal manometry, $n=5$ and 9 in the groups with normal gastric emptying and delayed gastric emptying respectively

$T_{50}$ Gastric emptying half time, LOS lower oesophageal sphincter, $n=$ number

Symptoms versus gastrointestinal dysmotility

Of the 16 different symptoms in the questionnaire, only abdominal fullness was significantly associated with delayed gastric emptying; it was found in all $(11 / 11)$ patients with delayed gastric emptying compared with only five out of nine patients without $(p=0.03) . T_{50}$ was significantly longer in patients with symptoms of abdominal fullness than in patients without (117 [42-255] vs 31 [19-59] $\min , p=0.04)$. No symptom predicted oesophageal dysmotility (data not shown).

Glucose homeostasis

The $\mathrm{HbA}_{1 \mathrm{c}}$ values indicated fair to good glycaemic control in the patients (Table 1). During the first hour after breakfast, subcutaneous glucose levels rose similarly in patients with and without delayed gastric emptying (Fig. 1a). Thereafter, glucose levels decreased in patients with delayed gastric emptying while continuing to rise in those without. Accordingly, the median glucose level was clearly lower in patients with delayed gastric emptying than in those without at $2.5 \mathrm{~h}$ after breakfast (9.1 [4.2-12.5] vs $14.3[11.2-17.7] \mathrm{mmol} / \mathrm{l} ; p<0.05)$. In seven of the 20 patients it was not possible to determine the AUC because of a temporary disconnection of the CGMS equipment after the first $2.5 \mathrm{~h}$. Patients with oesophageal dysmotility showed slow increments in glucose (abnormal vs normal manometry median glucose level; 11.6 [5.1-14.9] vs 14.4 [10.3-16.4] mmol/1, $1 \mathrm{~h}$ after breakfast, $p=0.16$ ) that continuously increased up to $4 \mathrm{~h}$ after breakfast whereas glucose decreased $2 \mathrm{~h}$ after breakfast in those with normal manometry (Fig. 1b). Similarly, the peak for glucose tended to be delayed in patients with abnormal oesophageal manometry vs those with normal oesophageal manometry (3.5 [1.8-4.9] vs $1.8[1.1-2.0] \mathrm{h} ; p=0.08)$.

There was no association between the $E / I$ ratio and glucose values after breakfast (data not shown). However, the glucose variation during the $72 \mathrm{~h}$ of monitoring was significantly larger in those with an abnormal $E / I$ ratio than in patients whose $E / I$ ratio was normal (coefficient of variation: 41 [46-49] vs 28 [27-34] \%; $p=0.008)$.

\section{Discussion}

This study showed that a feeling of abdominal fullness was associated with delayed gastric emptying. Patients with delayed gastric emptying showed decreased postprandial subcutaneous glucose concentrations, whereas patients with oesophageal dysmotility showed a tendency to delayed glucose absorption. Parasympathetic neuropathy was not associated with oesophageal dysmotility or abnormal gastric emptying but with increased variation in subcutaneous glucose concentrations. 


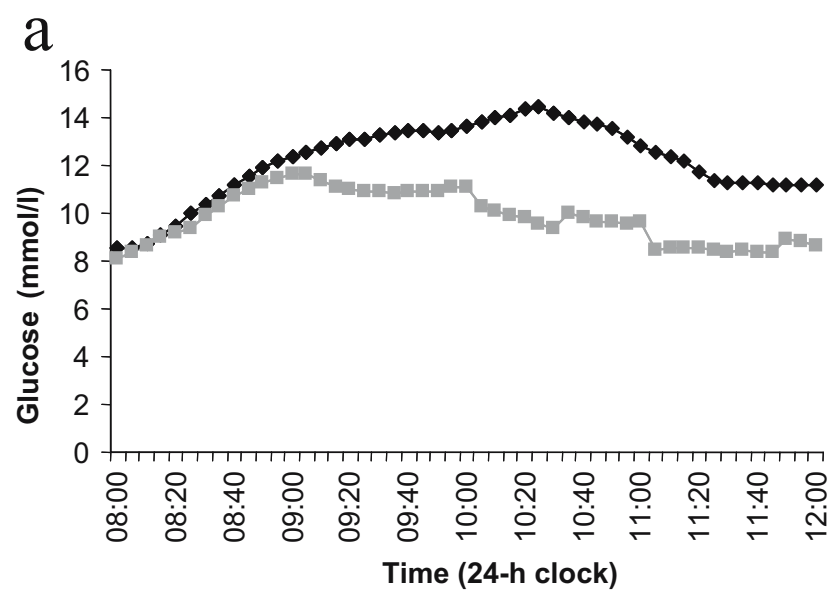

b

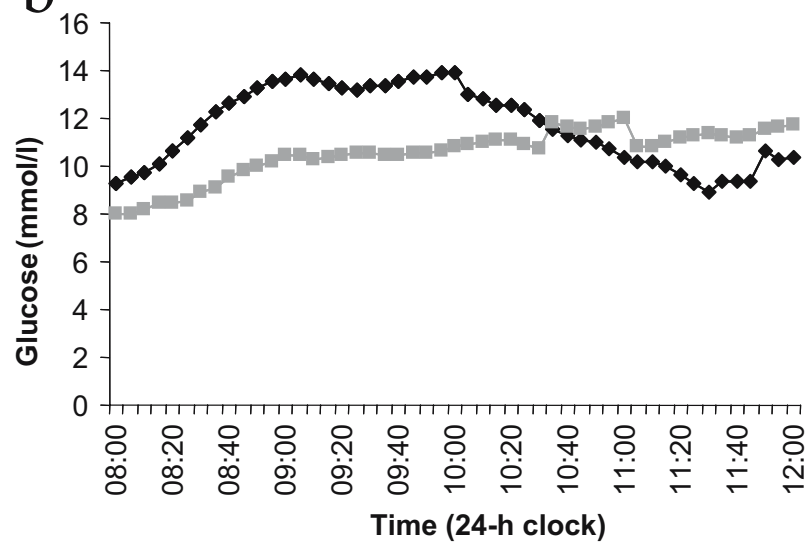

Fig. 1 Subcutaneous glucose concentrations after intake of a highfibre breakfast among patients with and without (a) delayed gastric emptying and (b) oesophageal dysmotility. There was a statistically significant difference in the median value in glucose concentration between patients with delayed vs normal gastric emptying $2.5 \mathrm{~h}$ $(p<0.05)$ after breakfast, and a trend for peak glucose values in patients with oesophageal dysmotility to be delayed $(p=0.08)$. Black symbols, patients with normal function; grey symbols, patients with abnormal function. Because of a temporary disconnection of the CGMS equipment between $2.5 \mathrm{~h}$ and $4 \mathrm{~h}$ after the test meal, values for seven of 20 patients were missing

Although it has been shown that variation in gastric emptying rate accounts for approximately $35 \%$ of the postprandial variance in glucose concentration in healthy volunteers and in patients with type 2 diabetes with normal gastric emptying [2], our study is the first to directly compare diabetic patients who show abnormal gastric emptying with diabetic patients whose gastric emptying is within the normal range.

The association between disturbed peristaltic oesophageal speed and disturbed gastric emptying is a novel finding [1]. Normal gastrointestinal motor function depends on a delicate balance between extrinsic and enteric nervous systems, smooth muscles and locally released transmitters [2]. Our finding of an obvious association between oesophageal delayed peristalsis and disturbed gastric emptying demonstrates for the first time a generally disturbed oesophageal and gastric motility in diabetic patients. This defect is most probably local (intrinsic) and not related to autonomic neuropathy. Our study did not provide any evidence that parasympathetic neuropathy (an abnormal $E / I$ ratio) was associated with oesophageal dysmotility, disturbed oesophageal propulsion or disturbed gastric emptying. Our study infers that decreased postprandial subcutaneous glucose concentration is a consequence of a proven upper gastrointestinal defect.

In this study, parasympathetic neuropathy was associated with increased variations in subcutaneous glucose levels. It was not associated with oesophageal and gastric function, and so it is unlikely that dysfunction of these organs was involved. Other mechanisms have to be considered to explain the disturbed glucose homeostasis in parasympathetic neuropathy. Impaired sensing of the portal venous-hepatic arterial glucose gradient in a rat model suggests that diabetic neuropathy involving intra-hepatic nerves could contribute to postprandial hyperglycaemia [3]. Surgical denervation of the liver and pancreas increases the glucagon response to oral glucose delivery in non-diabetic subjects [9]. Moreover, a muscarine cholinergic mechanism decreases hepatic glucose production in humans [10]. Parasympathetic neuropathy with impairment in this cholinergic system may enhance increased glucose production from the liver. Hence, different nervous mechanisms could explain why our diabetic patients with vagal neuropathy had a larger variation in subcutaneous glucose values than did patients without neuropathy.

In conclusion, this study shows that a low speed of oesophageal peristalsis is associated with slow gastric emptying in diabetic patients. This motility disturbance leads to delayed and decreased glucose uptake from the upper gastrointestinal tract. Parasympathetic neuropathy is not associated with this motility disturbance but with increased glucose fluctuations, possibly as a result of disturbed nervous humoral reflexes.

Acknowledgements We are grateful to A. Radelius and G. Willsteen for their skillful technical assistance in performing this study. This study was supported by grants from the Swedish Diabetes Association, the Ernhold Lundström Foundation, Research Funds of Malmö University Hospital, the Swedish Heart-Lung Foundation, the Albert Påhlsson Foundation, the Segerfalk Foundation, and the Swedish Medical Research Council. Mini Med Inc., Sweden, is thanked for providing the CGMS equipment.

Duality of interest None of the authors had any conflicting interests in connection with this study.

\section{References}

1. Horowitz M, Maddox AF, Wishart JM, Harding PE, Chatterton BE, Shearman DJ (1991) Relationships between oesophageal transit and solid and liquid gastric emptying in diabetes mellitus. Eur J Nucl Med 18:229-234

2. Rayner CK, Samsom M, Jones KL, Horowitz M (2001) Relationships of upper gastrointestinal motor and sensory function with glycemic control. Diabetes Care 24:371-381 
3. Stumpel F, Scholtka B, Jungermann K (1998) Impaired glucose sensing by intrahepatic, muscarinic nerves for an insulin-stimulated hepatic glucose uptake in streptozotocin-diabetic rats. FEBS Lett 436:185-188

4. Spechler SJ, Castell DO (2001) Classification of oesophageal motility abnormalities. Gut 49:145-151

5. Collins PJ, Horowitz M, Cook DJ, Harding PE, Shearman DJ (1983) Gastric emptying in normal subjects: a reproducible technique using a single scintillation camera and computer system. Gut 24:1117-1125

6. Hanson M, Lilja B (1987) Gastric emptying in smokers. Scand J Gastroenterol 22:1102-1104
7. Forsén A, Kangro M, Sterner G, et al (2004) A 14 year prospective study of autonomic nerve function in type 1 diabetic patients: associations with nephropathy. Diabet Med 21:852-858

8. Caplin NJ, O'Leary P, Bulsara M, Davis EA, Jones TW (2003) Subcutaneous glucose sensor values closely parallel blood glucose during insulin-induced hypoglycaemia. Diabet Med 20:238-241

9. Fabris SE, Thorburn A, Litchfield A, Proietto J (1996) Effect of parasympathetic denervation of liver and pancreas on glucose kinetics in man. Metabolism 45:987-991

10. Boyle PJ, Liggett SB, Shah SD, Cryer PE (1988) Direct muscarinic cholinergic inhibition of hepatic glucose production in humans. J Clin Invest 82:445-449 